\title{
Prevalence of tuberculosis infection in healthcare workers of the public hospital network in Medellín, Colombia: a Bayesian approach
}

\author{
J. OCHOA ${ }^{1 *}$, A. L. LEÓN ${ }^{1,2}$, I. C. RAMÍREZ ${ }^{3}$, C. M. LOPERA ${ }^{3}$, E. BERNAL $^{2}$ \\ AND M. P. ARBELÁEZ ${ }^{1}$ \\ ${ }^{1}$ Facultad Nacional de Salud Pública, 'Héctor Abad Gómez', Grupo de Epidemiología, Universidad de Antioquia, \\ Medellín, Colombia \\ ${ }^{2}$ Facultad de Medicina, Universidad de Antioquia, Medellín, Colombia \\ ${ }^{3}$ Escuela de Estadística, Universidad Nacional de Colombia, Sede Medellín
}

Received 22 June 2016; Final revision 29 November 2016; Accepted 7 December 2016; first published online 9 January 2017

\section{SUMMARY}

A latent tuberculosis infection (LTBI) prevalence survey was conducted using tuberculin skin test (TST) and Quantiferon test (QFT) in 1218 healthcare workers (HCWs) in Medellín, Colombia. In order to improve the prevalence estimates, a latent class model was built using a Bayesian approach with informative priors on the sensitivity and specificity of the TST. The proportion of concordant results (TST+,QFT + ) was $41 \%$ and the discordant results contributed $27 \%$. The marginal estimate of the prevalence $\mathrm{P}(\mathrm{LTBI}+)$ was $62 \cdot 1 \%$ [95\% credible interval $(\mathrm{CrI}) 53 \cdot 0-68 \cdot 2]$. The probability of LTBI+ given positive results for both tests was $99 \cdot 6 \%(95 \% \mathrm{CrI} 98 \cdot 1-99 \cdot 9)$. Sensitivity was 88.5 for TST and 74.3 for QFT, and specificity was $87 \cdot 8$ for TST and $97 \cdot 6$ for QFT. A high LTBI prevalence was found in HCWs with time-accumulated exposure in hospitals that lack control plans. In a context of intermediate tuberculosis (TB) incidence it is recommended to use only one test (either QFT or TST) in prevalence surveys or as pre-employment tests. Results will be useful to help implement TB infection control plans in hospitals where HCWs may be repeatedly exposed to unnoticed TB patients, and to inform the design of TB control policies.

Key words: Latent class model, occupational exposure, sensitivity, specificity.

\section{INTRODUCTION}

Several studies indicate that healthcare workers (HCWs), especially those who care for patients with pulmonary tuberculosis (TB) have a higher frequency of latent tuberculosis infection (LTBI) and active TB [1]. In the early twentieth century, for example, HCWs had a higher reported incidence of TB disease

\footnotetext{
* Author for correspondence: Dr J. Ochoa, Facultad Nacional de Salud Pública 'Héctor Abad Gómez', Universidad de Antioquia, Calle 62 No. 52-59, Medellín, Colombia.

(Email: jesus.ochoa@udea.edu.co)
}

than the general population [2]. The sustained reduction in the frequency of TB in industrialized countries decreased the interest in studying the risks workers have of becoming infected and getting sick. However, this interest increased in the 1990s when several outbreaks of TB in hospitals were described along with the importance of repeated, inadvertent exposures to patients without a TB diagnosis, and attention was again drawn to the increased risk of infection (LTBI) and disease in HCWs [2]. A recent systematic review indicated that the incidence of active $\mathrm{TB}$ in Latin American HCWs is 91 [interquartile range (IQR) 81-723] per 100000 , higher than the 
rate the authors use for comparison with the general population of those countries $(82 \times 100000$, IQR 28 223) [1].

LTBI is a complex condition difficult to diagnose, characterized by the presence of the immune response to infection by $M$. tuberculosis without clinical evidence of active TB. With respect to diagnostic methods of LTBI, the WHO recommends the tuberculin skin test (TST) or interferon- $\gamma$ release assay (IGRA) [3]. The latter is expensive and little studied in Colombia, but is being increasingly used because of its advantages compared to the TST; i.e. requires only a single visit, has comparable sensitivity and improved specificity in people with previous immunization with bacillus Calmette-Guérin (BCG), such as the case of the population of Colombian HCWs [4]. In Colombia, the performance of TST and IGRA has not been assessed in HCWs and the occupational risk of TB for HCWs remains mainly unaddressed in practice. Furthermore, there are no national or local guidelines to monitor HCWs and there is a lack of local knowledge on this subject in Colombia. In general, high-income countries include the diagnosis and treatment of LTBI in their plans to eliminate TB, have TB control plans in their hospitals and allocate resources for the regular monitoring of HCWs. This situation is very different in countries of intermediate and high incidence of TB, such as Colombia, where the control programme is centred on the passive detection and treatment of TB cases and lacks information on the prevalence of LTBI in HCWs and the political will to systematize and consolidate hospital TB control plans.

In Medellín, Colombia, the incidence of TB (all forms) in 2013 was 63.8 cases $/ 100000$ inhabitants, but rates above 100/100 000 have been recorded in some areas of the city (comunas) [5]. The delay in the diagnosis of pulmonary $\mathrm{TB}$ is problematic for the city (it may increase the repeated exposure of HCWs with $M$. tuberculosis) given that the median between the onset of symptoms and the start of treatment was 61 days in 2014 (IQR 32-105) [6].

A 5-year study by the National Epidemiological Surveillance System describes an increase in the reported active TB cases in HCWs for Colombia for 2010 and 2011 [7]. Antioquia - the province where Medellín is located, contributes $16 \%(n=84 / 532)$ of the total TB cases in the country. The public hospital network of Medellín (ESE Metrosalud) has nine hospitals and one administrative centre with a total of $1532 \mathrm{HCWs}$ (official census at beginning of the study). Although these institutions supervise most of the treatments for TB patients in the city and are usually the first point of contact between the respiratory patient and the health services, they lack institutional TB control plans: poor ventilation conditions, lack of resources for isolates and absence of periodic training of HCWs to prevent TB exposition and infection.

With the foregoing in mind, a prevalence survey of LTBI was conducted using TST and IGRA [QuantiFERON $^{\circledR}$-TB Gold In-Tube test (QFT); Cellestis-Qiagen, Australia] simultaneously in a population of HCWs (vaccinated with BCG at birth) from 10 institutions of the public hospital network of Medellín. Because both tests are imperfect due to the lack of a gold standard for the diagnosis of LTBI, we built a latent class model (LCM), which considers that the results of the prevalence survey are influenced by a latent common variable (true state of LTBI), which can not be measured directly [8]. The fitting of the LCM model was made using a Bayesian approach.

The LCM estimated the improved prevalence of LTBI in HCWs using TST and QFT results, and the sensitivity and specificity of both tests. This information will allow us to assess the usefulness of both tests to monitor HCWs in a context of limited resources and help design the policies to control nosocomial transmission of M. tuberculosis in Colombia.

\section{METHODS}

\section{Study design and participants}

A LTBI prevalence survey was conducted in a cohort of $1218 \mathrm{HCWs}$ from nine hospitals and the administrative centre of the public hospital network in the city of Medellín, Colombia, between 2013 and 2015.

We set out to study the totality of HCWs (clinical, administrative and support personnel). Of 1532 registered employees, 1218 voluntarily agreed to participate (Fig. 1). We report on the results of 1004 HCWs who received both the TST and QFT. The HCWs from one hospital and the administrative centre $(n=214)$ were not included in the analysis because they received only the QFT due to the world shortage of TST, including Tubersol ${ }^{\circledR}$ (PPD; Sanofi Pasteur Ltd, Canada) [9, 10], which affected the availability of the test in Colombia for 6 months.

We used TST and QFT simultaneously. Both tests are markers of exposure to M. tuberculosis and measure the body's immune response: TST produces a skin 


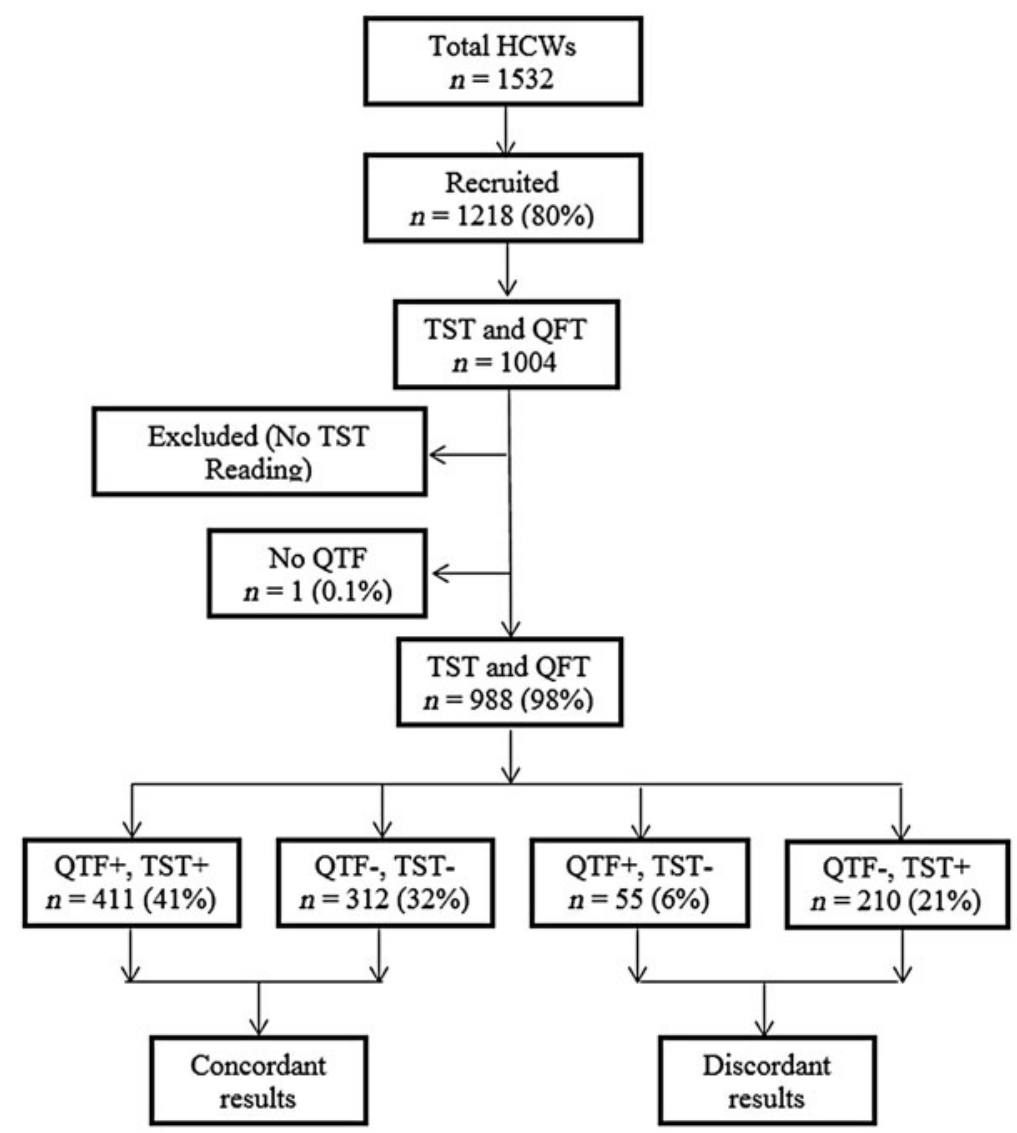

Fig. 1. Flow chart of study subjects. Prevalence of latent tuberculosis infection in healthcare workers (HCWs) of the public hospital network in Medellín, Colombia, 2013-2015.

induration on the site of application which is read in millimetres; QFT quantifies the production of interferon-gamma (IFN- $\gamma)$ by $\mathrm{T}$ cells [11]. The results of both tests are independent, although QFT may vary when performed $72 \mathrm{~h}$ after application of TST, and the sources of variability are different for each test [12].

\section{TST}

Five tuberculin units (Tubersol PPD) were used with each HCW. The application was made using the Mantoux method [13] by two trained professionals: one in the beginning of the study and a different one towards the end. We trained our personnel extensively to minimize bias. The measurement of induration was performed $72 \mathrm{~h}$ after application, and a result $\geqslant 10$ $\mathrm{mm}$ was considered positive. If the initial result was $<10 \mathrm{~mm}$, a second test was performed within a 1- to 3 -week interval to control for the 'booster effect' an increase in the induration in the absence of a new infection, caused by the recall of waned cell-mediated immunity [14]. The TST was performed immediately after sampling the blood for QFT.

\section{IGRA testing}

We used the QFT. Samples from HCWs were obtained by phlebotomy ( $1 \mathrm{ml}$ blood for each of the three tubes provided by the manufacturer). The tubes were shaken for $5 \mathrm{~s}$ and placed in an incubator transport (Cellestis) that guaranteed a temperature of $37^{\circ} \mathrm{C}$ until arrival at a certified laboratory. The results were presented according to the manufacturer's instructions with the specific software complement (QFT software v. 2·17, Cellestis). The test was considered positive if the results of IFN- $\gamma$ production minus those obtained in the null tube were $\geqslant 0.35 \mathrm{IU} / \mathrm{ml}$. The technician performing the QFT was blind to the results of the TST.

The demographic and occupational characteristics of the HCWs were described and the kappa statistic [15] for concordance between TST and QFT was calculated. Frequency histograms were prepared to verify 


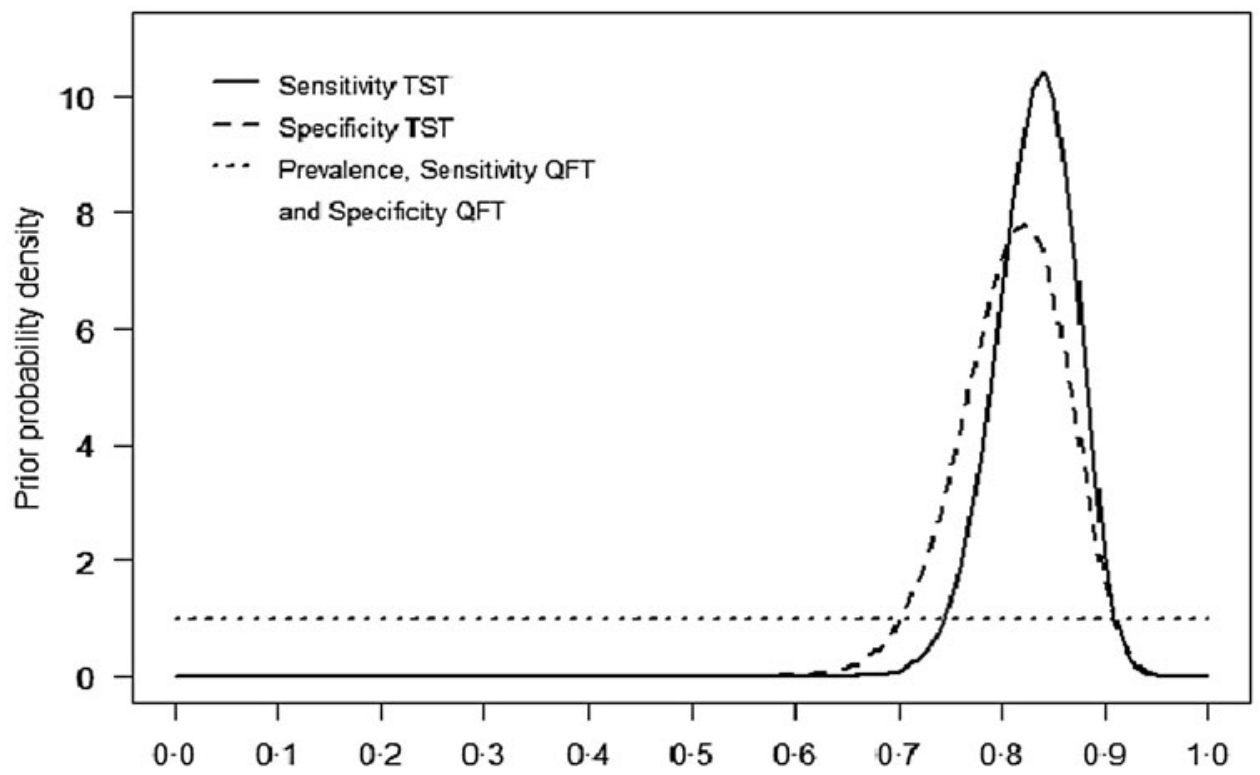

Fig. 2. Prior distributions used for the estimates. Prevalence of latent tuberculosis infection in healthcare workers of the public hospital network in Medellín, Colombia, 2013-2015.

the form of the distribution of the TST results by age and years of employment.

\section{Bayesian approach for the estimation of LCM}

We used the studies by Ling et al. [16] and Joseph et al. [8] as a reference and the following procedures for the construction of the LCM:

- We assigned 1 and 0 values to the positive and negative results, respectively, for both the TST and QFT. Frequencies for concordant and discordant results of the two tests were calculated (Fig. 1).

- We modelled the observed data using a multinomial distribution where the probabilities of the four combinations of the TST and QFT results can be expressed in terms of sensitivity and specificity and the prevalence of LTBI [equation (1) in the Technical Appendix].

- The LCM has the assumption of conditional independence between TST and QFT within the LTBI + and LTBI- groups. We did not control for dependence in the analysis but we are confident that the Bayesian LCM will allow for reasonable inferences [17].

- The LCM is not identifiable because there are five unknown parameters (the sensitivity and specificity of both tests and the prevalence) which exceed three degrees of freedom (four test combinations minus 1). To avoid this problem using a Bayesian approach, informative prior distributions were assigned for two of the model parameters (TST sensitivity and specificity).

- We obtained the percentiles of the prior distributions for the sensitivity and specificity of TST from an article by Pai et al. [18]. They worked with a cohort of 719 medical and nursing students and HCWs in India who underwent TST and QFT testing, and we used their LCM analysis results as prior distributions for our study. To estimate the sensitivity of the TST, a Beta distribution $(77 \cdot 8,15 \cdot 7)$ was used as informative prior distribution, whose $2.5 \%$ and $97.5 \%$ percentiles are 0.75 and 0.90 , respectively; and for the specificity of TST, a Beta distribution $(46 \cdot 3,10 \cdot 8)$ was used as prior distribution whose $2.5 \%$ and $97.5 \%$ percentiles are 0.70 and 0.90 , respectively (Fig. 2). We do not expect TST sensitivity/specificity to behave differently in our study, since the study by Pai et al. was also on HCWs and both India and Colombia administer BGC only once at birth.

- A relatively uninformative distribution such as the uniform distribution (Fig. 2) was used for the parameters of the model for which prior information was not available due to, as far as we know, a lack of Latin American studies on this subject (sensitivity and specificity for QFT). 
Table 1. Sociodemographic and occupational characteristics of the healthcare worker (HCW) population

\begin{tabular}{|c|c|c|c|c|c|c|c|c|c|c|}
\hline \multirow[b]{2}{*}{ Variables } & \multicolumn{2}{|c|}{$\begin{array}{l}\text { Total } \\
(N=988)\end{array}$} & \multicolumn{2}{|c|}{$\begin{array}{l}\text { QFT }+, \mathrm{TST}+ \\
(N=411 \\
41 \cdot 6 \%)\end{array}$} & \multicolumn{2}{|c|}{$\begin{array}{l}\text { QFT-,TST- } \\
(N=312 ; \\
31 \cdot 6 \%)\end{array}$} & \multicolumn{2}{|c|}{$\begin{array}{l}\text { QFT+,TST- } \\
(N=55 ; 5 \cdot 6 \%)\end{array}$} & \multicolumn{2}{|c|}{$\begin{array}{l}\text { QFT-,TST+ } \\
(N=210 \\
21 \cdot 3 \%)\end{array}$} \\
\hline & $n$ & $\%$ & $n$ & $\%$ & $n$ & $\%$ & $n$ & $\%$ & $n$ & $\%$ \\
\hline \multicolumn{11}{|l|}{ Sex } \\
\hline Female & 727 & $73 \cdot 6$ & 310 & $75 \cdot 4$ & 241 & $77 \cdot 2$ & 39 & $70 \cdot 9$ & 137 & $65 \cdot 2$ \\
\hline BCG vaccination status & 947 & $96 \cdot 7$ & 390 & $95 \cdot 8$ & 302 & $98 \cdot 1$ & 52 & $94 \cdot 6$ & 203 & $97 \cdot 1$ \\
\hline \multicolumn{11}{|l|}{ Education level } \\
\hline Illiterate/Elementary/High school & 147 & $14 \cdot 9$ & 83 & $20 \cdot 2$ & 31 & $9 \cdot 9$ & 9 & $16 \cdot 4$ & 24 & $11 \cdot 4$ \\
\hline Technical/Higher education/Other & 841 & $85 \cdot 1$ & 328 & $79 \cdot 8$ & 281 & $90 \cdot 1$ & 46 & $83 \cdot 6$ & 186 & $88 \cdot 6$ \\
\hline \multicolumn{11}{|c|}{ Socioeconomic strata (housing) } \\
\hline Low/Medium-low & 645 & $65 \cdot 3$ & 287 & $69 \cdot 8$ & 196 & $62 \cdot 8$ & 42 & $76 \cdot 4$ & 120 & $57 \cdot 1$ \\
\hline Medium-high/High & 343 & $34 \cdot 7$ & 124 & $30 \cdot 2$ & 116 & $37 \cdot 2$ & 13 & $23 \cdot 6$ & 90 & $42 \cdot 9$ \\
\hline \multicolumn{11}{|l|}{ Job type } \\
\hline Administrative & 242 & $24 \cdot 5$ & 100 & $24 \cdot 3$ & 67 & $21 \cdot 5$ & 15 & $27 \cdot 3$ & 60 & $28 \cdot 6$ \\
\hline Clínical/Para-clinical & 746 & $75 \cdot 5$ & 311 & $75 \cdot 7$ & 245 & $78 \cdot 5$ & 40 & $72 \cdot 7$ & 150 & $71 \cdot 4$ \\
\hline Sharing housing with a TB case & 55 & $5 \cdot 6$ & 33 & $8 \cdot 1$ & 15 & $4 \cdot 8$ & 1 & $1 \cdot 8$ & 6 & $2 \cdot 9$ \\
\hline Use of respiratory protection & 559 & $56 \cdot 6$ & 244 & $59 \cdot 4$ & 183 & $58 \cdot 7$ & 32 & $58 \cdot 2$ & 100 & $47 \cdot 6$ \\
\hline \multicolumn{11}{|l|}{ Residential area* } \\
\hline Low TB incidence & 749 & $78 \cdot 0$ & 287 & $71 \cdot 9$ & 255 & $84 \cdot 2$ & 39 & $70 \cdot 9$ & 168 & $82 \cdot 8$ \\
\hline Intermediate $\mathrm{TB}$ incidence & 195 & $20 \cdot 3$ & 105 & $26 \cdot 3$ & 43 & $14 \cdot 2$ & 15 & $27 \cdot 3$ & 32 & $15 \cdot 8$ \\
\hline \multirow[t]{2}{*}{ High TB incidence } & 16 & $1 \cdot 7$ & 7 & $1 \cdot 8$ & 5 & $1 \cdot 6$ & 1 & $1 \cdot 8$ & 3 & $1 \cdot 5$ \\
\hline & Median & IQR & Median & IQR & Median & IQR & Median & IQR & Median & IQR \\
\hline Age, years & 43 & $33-51$ & 44 & $36-52$ & 37 & $29-48$ & 47 & $37-52$ & 44 & $35-51$ \\
\hline Years of hospital employment & 14 & $6-23$ & 16 & $8-24$ & 10 & $4-21$ & 14 & $6-25$ & 16 & $8-24$ \\
\hline
\end{tabular}

BCG, Bacille Calmette-Guérin; IQR, interquartile range.

* The residential area of $28 \mathrm{HCWs}$ was unknown.

- Using the medians of age and years of employment (Table 1), the results of laboratory tests were processed for four groups of HCWs: age $<43$ years, age $\geqslant 43$ years, $<15$ years of employment, $\geqslant 15$ years of employment. The LCM results were presented for each group.

- Once the posterior distributions were obtained, we calculated the median and the credible intervals (CrIs) for prevalence, sensitivity and specificity for TST and QFT. In a Bayesian approach, the CrIs are the equivalent of the confidence intervals (CIs) used in the frequentist approach.

All the procedures described above were performed using OpenBUGS software, v. 3.2.3 revision 2012 (Bayesian inference Using Gibbs Sampling; Free Software Foundation, USA). The analysis presented here is the result of 20000 Markov chain Monte Carlo (MCMC) iterations using the multivariate forward updater algorithm with a thinning of five iterations and a burn-in of 10000 iterations. We checked the convergence of chains using the diagnostics tool provided by OpenBUGS (see the code in the Technical Appendix).

\section{Ethical considerations}

The authors assert that all the procedures contributing to this work comply with the ethical standards of the relevant national and institutional committees (Bioethics Review Committee of The National School of Public Health - University of Antioquia: C13255-161040 and the Management Committee at ESE Metrosalud) on human experimentation and with the Helsinki Declaration of 1975 , as revised in 2008. All the workers and managers of the healthcare institutions who agreed to participate gave their written informed consent (individual and institutional) accepting to take the survey, laboratory tests and clinical and X-ray follow-up as indicated in the protocols. The procedures to assess the presence of active TB were the estimation of comorbidity and the annual risk of active TB for all workers, using an algorithm developed by Menzies et al. [19] (The Online TST/ 
IGRA Interpreter, v. 3.0; www.tstin3d.com). Chest $\mathrm{X}$-rays were given to all workers with either one or both positive tests results, after which they were evaluated by an internist and/or pneumologist to establish individual prescriptions. TB smear and culture of sputum were given to respiratory symptomatics. In the case of LTBI, the national guidelines of Colombia postponed the decision on the systematic treatment and monitoring of LTBI in HCWs, probably following the conditional status of that recommendation in the WHO guidelines due to the "low quality of evidence' [20-22].

\section{RESULTS}

Some basic demographic and occupational characteristics are described in Table 1. The median age of the HCWs with positive concordant results (TST+, QFT+) was 43 (IQR 33-51) years, and for those with negative concordant results was 44 (IQR 3652 ) years. No differences in the history of BCG vaccination were found. Just over $75 \%$ of HCWs performed clinical and support activities and 98\% lived in areas of the city with low $(<50 \times 100000)$ and intermediate TB incidence (50-100/100000 inhabitants). Of the HCW respondents, 5.6\% had contact with active TB cases at their residence. These characteristics had about the same distribution in HCWs with discordant results. The concordance between TST and QFT was $73 \cdot 2 \%$ (kappa: $47 \cdot 1 \%$ 95\% CI 44·0-50·3).

Figure 1 summarizes the frequency of positive concordant (TST+,QFT + ), negative concordant (TST-, QFT-), and discordant (TST-,QFT+), (TST+,QFT-) results. Altogether, the discordant results had a frequency of $27 \%(n=265)$.

The frequency histograms for TST showed a bimodal distribution with assymetry to the left (coefficient of skewness: $-0 \cdot 3$ ) due to the number of HCWs who did not develop any induration, especially in those aged $<43$ years. The average induration was $9.6 \mathrm{~mm}$ (s.D. $=6 \cdot 0$ ). Figure 3 shows the histograms by age and years of employment, which had a similar distribution.

Figure 4 and Table 2 summarize the shape of the distribution and the posterior probabilities calculated by the model. The posterior probability that both tests are positive $\mathrm{P}(\mathrm{TST}+\mathrm{QFT}+)$ was $41.0 \%(95 \%$ CrI 38.0-44.0) and the prevalence obtained by the model $\mathrm{P}(\mathrm{LTBI}+)$ was $62 \cdot 1 \%(95 \% \mathrm{CrI} 53 \cdot 0-68 \cdot 2)$. The probability of having LTBI given positive results in both tests $\mathrm{P}(\mathrm{LTBI}+\mid \mathrm{TST}+, \mathrm{QFT}+)$ was $99 \cdot 6 \%(95 \%$
CrI 98·1-99.9), and for the negative results $\mathrm{P}(\mathrm{LTBI}+\mid$ TST-,QFT-) was 5.7\% (95\% CrI 2·5-9·2).

The posterior TST sensitivity was $88.5 \%(95 \% \mathrm{CrI}$ $85 \cdot 2-92 \cdot 1)$, and the specificity was $80 \cdot 8 \%(95 \% \mathrm{CrI}$ 68.9-89.6). For QFT, a posterior sensitivity of $74 \cdot 3 \%(95 \%$ CrI $67 \cdot 8-85 \cdot 6)$, and a specificity of $97 \cdot 6 \%$ (95\% CrI 91.6-99.9) were obtained.

The results of the LCM are presented by age and years of employment. Higher prevalences were obtained for the group of HCWs aged $\geqslant 43$ years compared to HCWs aged <43 years; however, the estimated probability of having LTBI $\mathrm{P}(\mathrm{LTBI}+\mid \mathrm{TST}+$, QFT+), the discordant results at the expense of QFT P(LTBI+|TST-,QFT+) and the sensitivity and specificity were similar by age group and years of employment (Table 2).

\section{DISCUSSION}

To our knowledge, this is the first study of LTBI in a HCW population in Colombia using TST and QTF simultaneously. Using the LCM, the probability of LTBI as measured by two tests (TST,$+ \mathrm{QFT}+)$ was $41 \cdot 0 \%$ and the prevalence was $62 \cdot 1 \%$. This prevalence was not surprising and fell within the range of the estimates for HCWs in low-/medium-income countries (33-79\%) [23]. In 2013 Nienhaus et al. [24] consolidated the results of LTBI prevalence in HCWs using TST and IGRA simultanously, and indicated that the prevalence of concordant results for medium-/high-income countries such as Spain was $30.0 \%$, Italy $21 \cdot 7 \%$, Portugal $30 \cdot 5 \%$, and Georgia $50 \cdot 8 \%$.

There are few studies of LTBI prevalence in HCWs using TST and IGRA simultaneously in countries of intermediate TB incidence and universal coverage of BCG vaccination at birth [25]. Nevertheless, a recent Colombian study [26] found a $66 \cdot 3 \%$ prevalence of LTBI in household contacts of TB cases in the city of Medellín, and $24 \cdot 3 \%$ in the general population. Despite the difficulty of comparing both studies due to the use of different laboratory tests, the prevalence of LTBI in HCWs in our study is similar that of the household contacts of active TB cases, and higher than that of the general population of the city.

The prevalence of LTBI and the fequency of discordant results in our study were similar to those found in countries of high TB incidence in the general population such as Georgia [27]: (TST+,QFT+: $50 \cdot 2 \%)(\mathrm{TST}+$,QFT-: $16 \cdot 6 \%)$ (TST-,QFT+: $3 \cdot 8 \%$ ) or India [28] where prevalences of $50 \%$ were found for either test, and $30 \%$ for both tests in young HCWs 

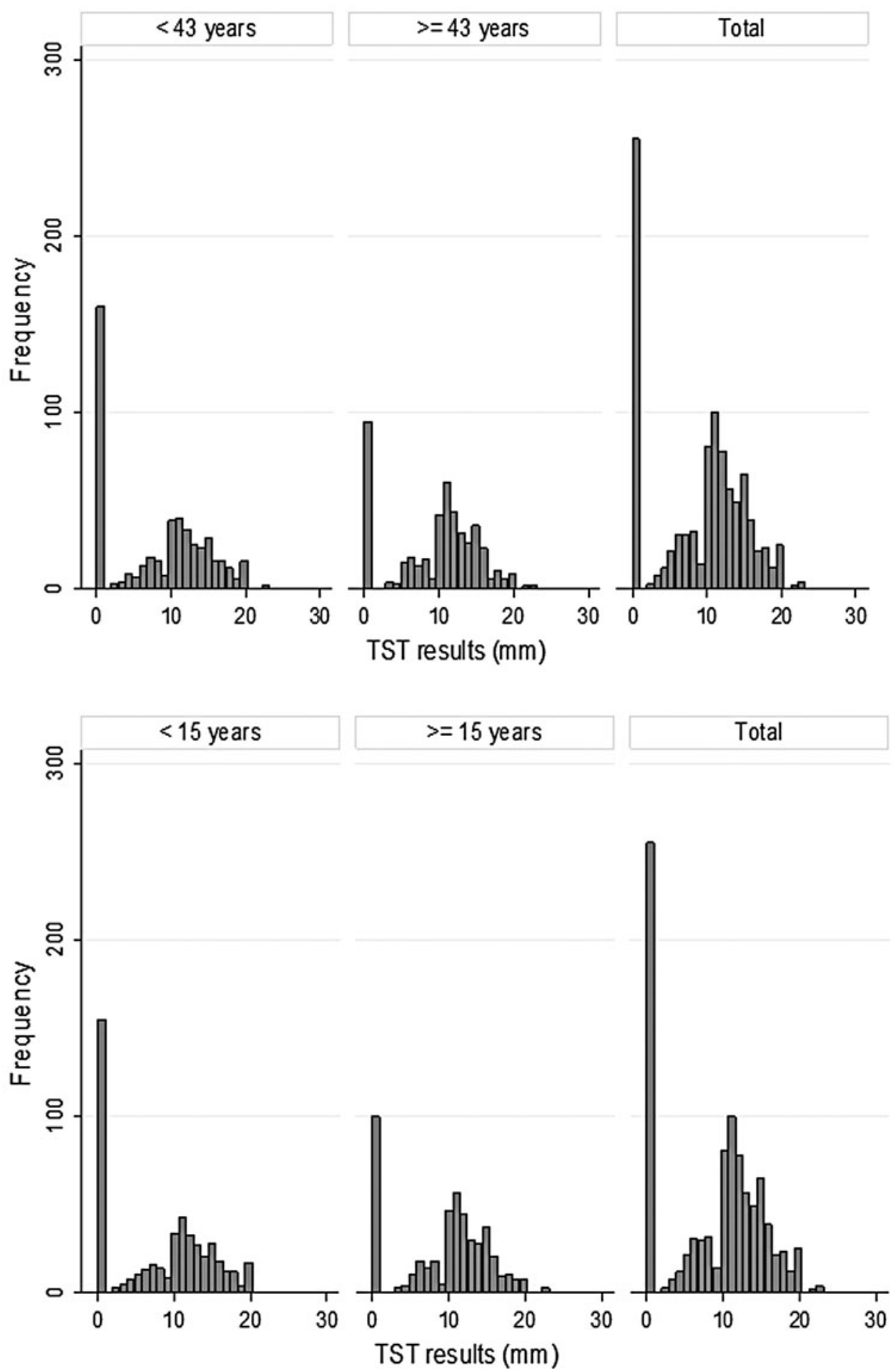

Fig. 3. Frequency histograms of the distribution of the tuberculin skin test (TST) results by age and years of employment.

(median age 22 years). The interpretation of discordant results (TST+,QFT-) (TST-,QFT+) is complex due to the rooted tendency of considering them as TST false positives caused by cross-reactivity with BCG and/or the infection with non-TB mycobacterias
[18]. Moreover, although we trained our personnel extensively, some two-reader bias is still possible as described by Menzies [14] and this may impact the proportion of discordant results. It is also necessary to consider other sources of variability of QFT such 


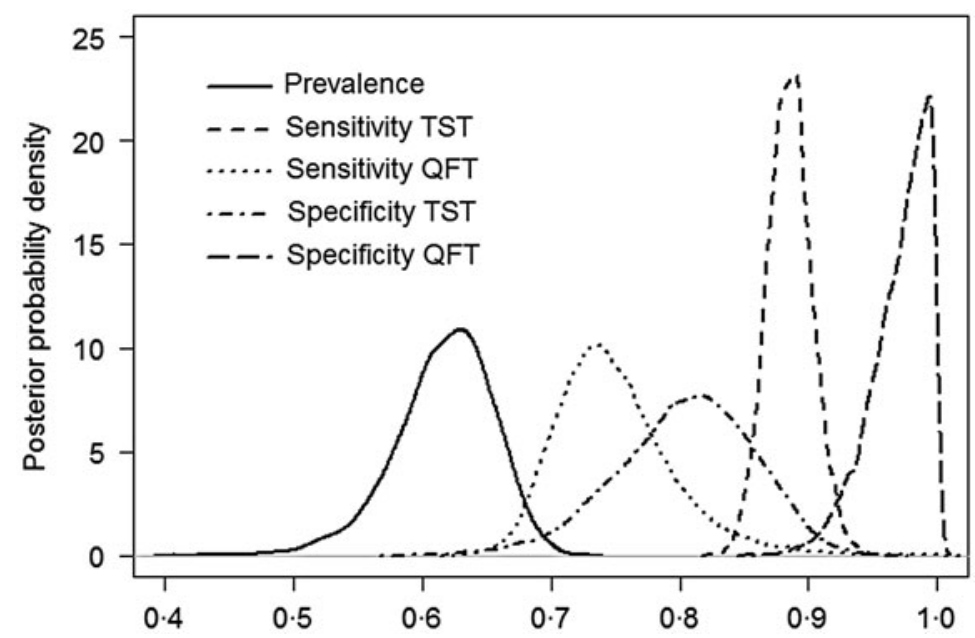

Fig. 4. Posterior distribution estimates for the parameters of the model (prevalence, sensitivity, specificity). Prevalence of latent tuberculosis infection in healthcare workers of the public hospital network in Medellín, Colombia, $2013-2015$.

as the drawing, transport and processing of samples [29], and the possibility that the HCWs with QFTresults in our study could correspond to false negatives or reversions [30].

Our results indicate the importance of further studying the weight of occupational and nonoccupational (community) exposure in LTBI in HCWs in Colombia. We found a group of $21 \%$ of HCWs with discordant (TST+,QFT-) results, $82 \cdot 8 \%$ of whom lived in areas of the city with low TB incidence in the population and had a median age of 44 (IQR 35-51) years. These results are threefold those of Zwerling et al. [31] who found $8.6 \%$ of discordant results (TST+,QFT-) in a low-incidence country, associated with the non-occupational exposure and the total number of years of hospital employment.

Several approaches (frequentist and Bayesian) have been described to estimate the prevalence of a disease and the accuracy of diagnostic tests in the absence of a gold standard. The robust estimates obtained by the LCM in our study allowed for a more accurate approach to quantifying the prevalence of LTBI and considered the uncertainty of the sensitivity and specificity of the tests in a population of HCWs not previously studied and vaccinated with BCG. The operational features of TST and QFT obtained in our study are broadly consistent with those obtained by Ling and Pai in HCWs in India $[16,18]$.

One limitation of our study is the current lack of information about the performance of the tests in the group of HCWs with negative concordant results a year later ('annual conversion'), as well as the lack of information on reversions. Thus, obtaining more informative data in the future would require serial and periodic screening [32]. Moreover, although the quality of prior information may affect LCM results is a known criticism [16], we still believe that we have used the best available information on sensitivity and specificity of TST and QFT tests from a study in $\mathrm{HCWs}$ vaccinated with $\mathrm{BCG}$ at birth.

Another limitation is that our study does not provide objective data on the control of conditional dependence of both tests. If the TST and QFT were positively correlated, the sensitivity and specificity may be biased. Yet, some authors have found that the magnitude of the bias is mitigated by the Bayesian LCM that adjusts for dependence, and even those models which ignore the dependence allow for reasonable inferences [16, 17, 33].

There are challenges to measuring the exposure gradient in a prevalence study such as this one and we used both age and years of employment as stratification variables. Besides the great variation in the definition of the exposure groups [34], the time of employment of HCWs does not account for their community exposure to TB infection, which may be significant in intermediate-/high-incidence cities such as Medellín. The HCWs' age, on the other hand, reflects the cumulative exposure (occupational plus community exposure) [35], and is easier to use as a means of prioritizing populations at risk in institutional TB control programmes.

Our study is the beginning of a journey along a road not travelled in Colombia thus far. We have previously published a simulation of the risk of infection in hospitals where the HCWs in our study come from [36] and we 
Table 2. Predictive posterior probabilities: prevalence, sensitivity and specificity, and probabilities of concordant and discordant results for healthcare workers ( $H C W s$ ) and for age groups $<43$ and $\geqslant 43$ years. Prevalence of latent tuberculosis infection (LTBI) in HCWs of the public hospital network in Medellin, Colombia, 2013-2015

\begin{tabular}{|c|c|c|c|c|c|c|}
\hline \multirow[b]{3}{*}{ Variable } & \multicolumn{3}{|c|}{ Age $(<43$ and $\geqslant 43$ years $)$} & \multicolumn{3}{|c|}{$\begin{array}{l}\text { Years of hospital employment } \\
(<15 \text { and } \geqslant 15 \text { years })\end{array}$} \\
\hline & \multirow[b]{2}{*}{ Median, $\%$} & \multicolumn{2}{|c|}{$95 \% \mathrm{CrI}$} & \multirow[b]{2}{*}{ Median, $\%$} & \multicolumn{2}{|c|}{$95 \% \mathrm{CrI}$} \\
\hline & & $2 \cdot 50 \%$ & $97 \cdot 50 \%$ & & $2 \cdot 50 \%$ & $97 \cdot 50 \%$ \\
\hline Probability $(\mathrm{TST}+, \mathrm{QFT}+)$ & $41 \cdot 04$ & $38 \cdot 02$ & $44 \cdot 04$ & $41 \cdot 04$ & $38 \cdot 02$ & $44 \cdot 04$ \\
\hline$<43$ & $36 \cdot 13$ & $32 \cdot 20$ & $40 \cdot 39$ & $36 \cdot 75$ & $32 \cdot 82$ & $40 \cdot 92$ \\
\hline$\geqslant 43$ & $44 \cdot 86$ & $40 \cdot 70$ & $49 \cdot 13$ & $44 \cdot 56$ & $40 \cdot 43$ & $48 \cdot 85$ \\
\hline Probability (TST+,QFT-) & $21 \cdot 23$ & $18 \cdot 78$ & $23 \cdot 89$ & $21 \cdot 23$ & $18 \cdot 78$ & $23 \cdot 89$ \\
\hline$<43$ & $20 \cdot 41$ & $17 \cdot 03$ & $24 \cdot 05$ & $19 \cdot 20$ & $15 \cdot 97$ & $22 \cdot 84$ \\
\hline$\geqslant 43$ & $22 \cdot 12$ & $18 \cdot 71$ & $25 \cdot 91$ & $23 \cdot 53$ & $19 \cdot 92$ & $27 \cdot 37$ \\
\hline Probability (TST-,QFT+) & $6 \cdot 15$ & $4 \cdot 86$ & $7 \cdot 66$ & $6 \cdot 15$ & $4 \cdot 86$ & $7 \cdot 66$ \\
\hline$<43$ & $5 \cdot 77$ & $4 \cdot 17$ & $7 \cdot 75$ & $6 \cdot 70$ & $4 \cdot 93$ & $8 \cdot 87$ \\
\hline$\geqslant 43$ & $7 \cdot 42$ & $5 \cdot 53$ & $9 \cdot 73$ & $6 \cdot 55$ & $4 \cdot 77$ & $8 \cdot 67$ \\
\hline Probability (TST-,QFT-) & $31 \cdot 51$ & $28 \cdot 63$ & $34 \cdot 44$ & $31 \cdot 51$ & $28 \cdot 63$ & $34 \cdot 44$ \\
\hline$<43$ & $37 \cdot 57$ & $33 \cdot 31$ & $41 \cdot 92$ & $37 \cdot 24$ & $32 \cdot 98$ & $41 \cdot 50$ \\
\hline$\geqslant 43$ & $25 \cdot 43$ & $21 \cdot 71$ & $29 \cdot 42$ & $25 \cdot 24$ & $21 \cdot 51$ & $29 \cdot 31$ \\
\hline Prevalence (LTBI+) & $62 \cdot 06$ & $53 \cdot 03$ & $68 \cdot 25$ & $62 \cdot 06$ & $53 \cdot 03$ & $68 \cdot 25$ \\
\hline$<43$ & $54 \cdot 15$ & $43 \cdot 49$ & $62 \cdot 29$ & $54 \cdot 27$ & $43 \cdot 64$ & $62 \cdot 47$ \\
\hline$\geqslant 43$ & $69 \cdot 82$ & $61 \cdot 08$ & $76 \cdot 44$ & $70 \cdot 32$ & $61 \cdot 83$ & $76 \cdot 86$ \\
\hline Prevalence $(\mathrm{LTBI}+\mid \mathrm{TST}+, \mathrm{QFT}+)$ & $99 \cdot 59$ & $98 \cdot 07$ & $99 \cdot 98$ & $99 \cdot 59$ & $98 \cdot 07$ & $99 \cdot 98$ \\
\hline$<43$ & $99 \cdot 54$ & $97 \cdot 71$ & $99 \cdot 98$ & $99 \cdot 37$ & $97 \cdot 02$ & $99 \cdot 97$ \\
\hline$\geqslant 43$ & $99 \cdot 50$ & $97 \cdot 56$ & $99 \cdot 98$ & $99 \cdot 62$ & $98 \cdot 09$ & $99 \cdot 99$ \\
\hline Prevalence (LTBI+|TST+,QFT-) & $66 \cdot 65$ & $34 \cdot 30$ & $84 \cdot 33$ & $66 \cdot 65$ & $34 \cdot 30$ & $84 \cdot 33$ \\
\hline$<43$ & $57 \cdot 94$ & $17 \cdot 91$ & $80 \cdot 86$ & $55 \cdot 84$ & $14 \cdot 31$ & $80 \cdot 01$ \\
\hline$\geqslant 43$ & $75 \cdot 30$ & $49 \cdot 74$ & $88 \cdot 91$ & $76 \cdot 91$ & $52 \cdot 05$ & $89 \cdot 61$ \\
\hline Prevalence (LTBI+|TST-,QFT+) & $88 \cdot 18$ & $58 \cdot 13$ & $99 \cdot 47$ & $88 \cdot 18$ & $58 \cdot 13$ & $99 \cdot 47$ \\
\hline$<43$ & $87 \cdot 19$ & $56 \cdot 14$ & $99 \cdot 44$ & $84 \cdot 57$ & $51 \cdot 27$ & $99 \cdot 28$ \\
\hline$\geqslant 43$ & $86 \cdot 71$ & $55 \cdot 06$ & $99 \cdot 41$ & $88 \cdot 70$ & $58 \cdot 67$ & $99 \cdot 52$ \\
\hline Prevalence (LTBI+|TST-,QFT-) & $5 \cdot 67$ & $2 \cdot 51$ & $9 \cdot 17$ & $5 \cdot 67$ & $2 \cdot 51$ & $9 \cdot 17$ \\
\hline$<43$ & $4 \cdot 11$ & $1 \cdot 09$ & $7 \cdot 99$ & $4 \cdot 17$ & $0 \cdot 92$ & $8 \cdot 29$ \\
\hline$\geqslant 43$ & $8 \cdot 95$ & $4 \cdot 58$ & $15 \cdot 32$ & $8 \cdot 87$ & $4 \cdot 55$ & $15 \cdot 09$ \\
\hline Sensitivity (TST+|LTBI+) & $88 \cdot 55$ & $85 \cdot 22$ & $92 \cdot 06$ & $88 \cdot 55$ & $85 \cdot 22$ & $92 \cdot 06$ \\
\hline$<43$ & $88 \cdot 09$ & $83 \cdot 75$ & 91.98 & $86 \cdot 94$ & $82 \cdot 44$ & $91 \cdot 40$ \\
\hline$\geqslant 43$ & $87 \cdot 72$ & $83 \cdot 60$ & $91 \cdot 69$ & $88 \cdot 76$ & $84 \cdot 76$ & $92 \cdot 29$ \\
\hline Sensitivity (QFT+|LTBI+) & $74 \cdot 34$ & $67 \cdot 85$ & $85 \cdot 59$ & $74 \cdot 34$ & $67 \cdot 85$ & $85 \cdot 59$ \\
\hline$<43$ & $75 \cdot 47$ & $65 \cdot 79$ & $91 \cdot 42$ & $77 \cdot 48$ & $67 \cdot 59$ & $93 \cdot 49$ \\
\hline$\geqslant 43$ & $72 \cdot 97$ & $66 \cdot 24$ & $81 \cdot 75$ & $71 \cdot 25$ & $64 \cdot 40$ & $80 \cdot 05$ \\
\hline Specificity (TST-|LTBI-) & $80 \cdot 77$ & $68 \cdot 94$ & $89 \cdot 62$ & $80 \cdot 77$ & $68 \cdot 94$ & $89 \cdot 62$ \\
\hline$<43$ & $80 \cdot 73$ & $69 \cdot 88$ & $89 \cdot 56$ & $80 \cdot 77$ & $69 \cdot 95$ & $89 \cdot 56$ \\
\hline$\geqslant 43$ & $80 \cdot 75$ & $69 \cdot 42$ & $89 \cdot 51$ & $80 \cdot 90$ & $68 \cdot 94$ & $89 \cdot 77$ \\
\hline Specificity (QFT-|LTBI-) & $97 \cdot 63$ & $91 \cdot 65$ & $99 \cdot 89$ & $97 \cdot 63$ & $91 \cdot 65$ & $99 \cdot 89$ \\
\hline$<43$ & $98 \cdot 02$ & $92 \cdot 51$ & $99 \cdot 92$ & $97 \cdot 22$ & $90 \cdot 59$ & $99 \cdot 87$ \\
\hline$\geqslant 43$ & $95 \cdot 97$ & $86 \cdot 04$ & $99 \cdot 82$ & $96 \cdot 93$ & $88 \cdot 44$ & $99 \cdot 87$ \\
\hline
\end{tabular}

CrI, Credible interval.

now present the results for each of them. We consider it necessary to widen this study to include HCWs in healthcare centres (primary care) given that they are more likely to encounter unnoticed TB patients.

During the course of the present research we observed encouraging changes in the attitude of HCWs. Some of the hospitals started conducting diagnostic and early isolation activities (administrative controls), which although not the goal of the present research (effect not measured), may be attributed to the hospitalis participation in the study: the effect of the project's follow-up of hospital services, the administration of 
laboratory tests to HCWs and the construction of the project web page (http://www.epiteorica.com/index. php/tuberculosis-en-hospitales). Assessing the efficacy of these administrative controls would require further studies and measurement of the annual conversion rate of HCWs. In the meantime, a possibility for strenghtening these changes and implementing local TB control plans could be the adaptation of the FAST strategy: 'Find cases Actively by cough surveillance and rapid molecular sputum testing, Separate safely, and Treat effectively based on rapid drug susceptibility testing (DST)' with the goal of reducing time exposure to unnoticed TB patients [37].

In conclusion, our results indicate that a two-test strategy would not be useful in Colombia for the diagnosis of LTBI in HCWs. Although using two tests may improve the sensitivity, the clinical and epidemiological uncertainty generated by the discordant results would make prevalence surveys and pre-employment tests more costly and difficult to interpret. This reinforces the need for developing national guidelines that support the systematic monitoring of Colombian HCWs with a single test, either as a pre-employment test or in the assessment of the prevalence of LTBI in healthcare institutions. Although the Ministry of Health guidelines on TB recommend the use of TST as the test of choice in Colombia [20], it is necessary to conduct local cost-benefit studies to compare with QFT.

\section{ACKNOWLEDGEMENTS}

The authors thank the funding agencies and the directors and staff of the ESE Metrosalud. In particular, we thank all the HCWs who participated in the study. We also express our special thanks for the collaboration of Fernando Montes, Rita Almanza, Yuly Ortiz, Lisandra Arango, Doracelly Hincapié, Sara París, Sergio Yañez, María Cristina Hoyos, Hernán Sepúlveda, Silvia Echeverri and Leonardo Uribe.

This work was funded by the University of Antioquia (The Sustainability Strategy), ESE Metrosalud, Medellín Health Department (Agreement No. 4600042433), and by the Administrative Department of Science and Innovation of Colombia (COLCIENCIAS code: 111556934195).

\section{DECLARATION OF INTEREST}

None.

\section{REFERENCES}

1. Baussano I, et al. Tuberculosis among health care workers. Emerging Infectious Diseases 2011; 17: 488-494.

2. Sepkowitz KA. Tuberculosis and the health care worker: a historical perspective. Annals of Internal Medicine 1994; 120: 71-79.

3. Pai M, Sotgiu G. Diagnostics for latent TB infection: incremental, not transformative progress. European Respiratory Journal 2016; 47: 704-706.

4. Zwerling A, et al. The BCG World Atlas: a database of global BCG vaccination policies and practices. PLoS Medicine 2011; 8: e1001012.

5. Almanza R, et al. Health Secretary Medellín. Epidemiological profile tuberculosis Medellín 2013. Epidemiological Bulletin 3, 2014 (https://www.Medellín.gov.co/ irj/go/km/docs/pccdesign/SubportaldelCiudadano_2/ PlandeDesarrollo_0_19/Publicaciones/Shared\%20Content/ Boletines $/$ Bolet $\%$ C3 $\%$ ADn $\% 20$ epidemiol $\%$ C3 $\%$ B3gico $\%$ 203\%20de \%202014-Tuberculosis.pdf).

6. Rodriguez DA, et al. Monitoring delays in diagnosis of pulmonary tuberculosis in eight cities in Colombia. Pan American Journal of Public Health 2016; 39: 12-18.

7. Castillo D, Fadul S. Tuberculosis in health personnel, Colombia, 2008 to 2012. Bogotá, Colombia: National Epidemiological Fortnightly Report 2013; 2: 12-30.

8. Joseph L, Gyorkos TW, Coupal L. Bayesian estimation of disease prevalence and the parameters of diagnostic tests in the absence of a gold standard. American Journal of Epidemiology 1995; 141: 263-272.

9. Tebruegge M, et al. Shortage of purified protein derivative for tuberculosis testing. Lancet 2014; 384: 2026.

10. Anon. National shortage of purified-protein derivative tuberculin products. Morbidity and Mortality Weekly Report 2013; 62: 312.

11. Pai M, Rodrigues C. Management of latent tuberculosis infection: an evidence-based approach. Lung India 2015; 32: 205-207.

12. Zyl-Smit RNv, et al. Within-subject variability and boosting of T-cell interferon- $\gamma$ responses after tuberculin skin testing. American Journal of Respiratory and Critical Care Medicine 2009; 180: 49-58.

13. Lee E, Holzman RS. Evolution and current use of the tuberculin test. Clinical Infectious Diseases 2002; 34: 365-370.

14. Menzies D. Interpretation of repeated tuberculin tests. American Journal of Respiratory and Critical Care Medicine 1999; 159: 15-21.

15. Fleisss J. Statistical Methods for Rates and Proportions. New York: Wiley, 1981.

16. Ling D, et al. A Bayesian framework for estimating the incremental value of a diagnostic test in the absence of a gold standard. BMC Medical Research Methodology 2014; 14: 67.

17. Wang Z, Dendukuri N, Joseph L. Understanding the effects of conditional dependence in research studies involving imperfect diagnostic tests. Statistics in Medicine 2016. doi:10.1002/sim.7148.

18. Pai M, et al. Improving the estimation of tuberculosis infection prevalence using T-cell-based assay and 
mixture models. International Journal of Tuberculosis and Lung Disease 2008; 12: 895-902.

19. Menzies D, et al. Thinking in three dimensions: a webbased algorithm to aid the interpretation of tuberculin skin test results. International Journal of Tuberculosis and Lung Disease 2008; 12: 498-505.

20. Colombia. Ministry of Health and Social Protection. Adjustments to the current guidelines for the programmatic management of Tuberculosis and Leprosy: Technical Annex 3: Guidelines and recommendations of the Advisory National Committee for the diagnosis and treatment of latent tuberculosis infection 2015 (https://www.minsalud.gov.co/sites/rid/Lists/Biblioteca Digital/RIDE/DE/DIJ/circular-externa-0007-de-2015.pdf).

21. World Health Organization. Guidelines on the Management of Latent Tuberculosis Infection. Geneva: World Health Organization, 2015.

22. Getahun H, et al. Management of latent Mycobacterium tuberculosis infection: WHO guidelines for low tuberculosis burden countries. European Respiratory Journal 2015; 46: 1563-1576.

23. Joshi R, et al. Tuberculosis among health-care workers in low- and middle-income countries: a systematic review. PLoS Medicine 2006; 3: e494.

24. Nienhaus A, et al. IFN- $\gamma$ release assay versus tuberculin skin test for monitoring TB infection in healthcare workers. Expert Review of Anti-infective Therapy 2013; 11: 37-48.

25. Lamberti M, et al. Tuberculin skin test and Quantiferon test agreement and influencing factors in tuberculosis screening of healthcare workers: a systematic review and meta-analysis. Journal of Occupational Medicine and Toxicology 2015; 10: 1-13.

26. del Corral $\mathbf{H}$, et al. IFN $\gamma$ response to Mycobacterium tuberculosis, risk of infection and disease in household contacts of tuberculosis patients in Colombia. PLoS ONE 2009; 4: e8257.

27. A. Whitaker $\mathbf{J}$, et al. Prevalence and incidence of latent tuberculosis infection in Georgian healthcare workers. PLoS ONE 2013; 8: e58202.

28. Pai M, et al. Mycobacterium tuberculosis infection in health care workers in rural India. Journal of the American Medical Association 2005; 293: 2746-2755.

29. Pai M, et al. Gamma interferon release assays for detection of Mycobacterium tuberculosis infection. Clinical Microbiology Reviews 2014; 27: 3-20.

30. Dorman SE, et al. Interferon-gamma release assays and tuberculin skin testing for diagnosis of latent tuberculosis infection in healthcare workers in the United States. American Journal of Respiratory and Critical Care Medicine 2014; 189: 77-87.

31. Zwerling A, et al. TB screening in Canadian health care workers using interferon-gamma release assays. PLoS ONE 2012; 7: e43014.

32. Mazurek GH, et al. Updated guidelines for using interferon gamma release assays to detect Mycobacterium tuberculosis infection - United States, 2010. Morbidity and Mortality Weekly Report 2010; 59: 1-25.

33. Torrance-Rynard VL, Walter SD. Effects of dependent errors in the assessment of diagnostic test performance. Statistics in Medicine 1997; 16: 2157-2175.
34. Trajman A, Steffen RE, Menzies D. Interferon-gamma release assays versus tuberculin skin testing for the diagnosis of latent tuberculosis infection: an overview of the evidence. Pulmonary Medicine 2013; 2013: 11.

35. Menzies D, Joshi R, Pai M. Risk of tuberculosis infection and disease associated with work in health care settings. International Journal of Tuberculosis and Lung Disease 2007; 11: 593e605.

36. Ochoa J, et al. Simulation of risk of tuberculosis infection in healthcare workers in hospitals of an intermediate incidence country. Epidemiology and Infection 2015; 143: 2639-2647.

37. Barrera E, Livchits V, Nardell E. F-A-S-T: a refocused, intensified, administrative tuberculosis transmission control strategy. International Journal of Tuberculosis and Lung Disease 2015; 19: 381-384.

\section{TECHNICAL APPENDIX}

We used the multinomial distribution where the probabilities of the four combinations of the two test results can be expressed in terms of the sensitivity and specificity, and the prevalence of LTBI. Thus:

$n_{i j} \sim \operatorname{multinomial}\left(\pi_{i j}, N\right), i, j=0,1$ and $N=\sum_{i, j} n_{i, j}$,

where $n_{i j}$ represents the number of individuals classified in the status $i$ of the standard test and the status $j$ of the new test. The values ' 0 ' and ' 1 ' represent that the test was negative and positive respectively.

The values of $\pi_{i j}$ are given by:

$$
\begin{aligned}
\pi_{11}= & P\left(\mathrm{LTBI}^{+}\right) P\left(\mathrm{TST}^{+} \mid \mathrm{LTBI}^{+}\right) P\left(\mathrm{QFT}^{+} \mid \mathrm{LTBI}^{+}\right) \\
+ & {\left[1-P\left(\mathrm{LTBI}^{+}\right)\right]\left[1-P\left(\mathrm{TST}^{-} \mid \mathrm{LTBI}^{-}\right)\right] } \\
& {\left[1-P\left(\mathrm{QFT}^{-} \mid \mathrm{LTBI}^{-}\right)\right] } \\
\pi_{10}= & P\left(\mathrm{LTBI}^{+}\right) P\left(\mathrm{TST}^{+} \mid \mathrm{LTBI}^{+}\right) \\
& {\left[1-P\left(\mathrm{QFT}^{+} \mid \mathrm{LTBI}^{+}\right)\right]+\left[1-P\left(\mathrm{LTBI}^{+}\right)\right] } \\
& {\left.\left[1-P\left(\mathrm{TST}^{-} \mid \mathrm{LTBI}^{-}\right)\right] P\left(\mathrm{QFT}^{-} \mid \mathrm{LTBI}^{-}\right)\right) } \\
\pi_{01}= & P\left(\mathrm{LTBI}^{+}\right)\left[1-P\left(\mathrm{TST}^{+} \mid \mathrm{LTBI}^{+}\right)\right] \\
& P\left(\mathrm{QFT}^{+} \mid \mathrm{LTBI}^{+}\right)+\left[1-P\left(\mathrm{LTBI}^{+}\right)\right] \\
& P\left(\mathrm{TST}^{-} \mid \mathrm{LTBI}^{-}\right)\left[1-P\left(\mathrm{QFT}^{-} \mid \mathrm{LTB}^{-}\right)\right] \\
\pi_{00}= & P\left(\mathrm{LTBI}^{+}\right)\left[1-P\left(\mathrm{TST}^{+} \mid \mathrm{LTBI}^{+}\right)\right] \\
& {\left[1-P\left(\mathrm{QFT}^{+} \mid \mathrm{LTBI}^{+}\right)\right]+\left[1-P\left(\mathrm{LTBI}^{+}\right)\right] } \\
& P\left(\mathrm{TST}^{-} \mid \mathrm{LTBI}^{-}\right) P\left(\mathrm{QFT}^{-} \mid \mathrm{LTBI}^{-}\right)
\end{aligned}
$$

where:

$P\left(\mathrm{LTBI}^{+}\right)$is the prevalence of LTBI,

$P\left(\mathrm{TST}^{+} \mid \mathrm{LTBI}^{+}\right)$is the sensitivity of TST, $P\left(\mathrm{QFT}^{+} \mid \mathrm{LTBI}^{+}\right)$is the sensitivity of $\mathrm{QFT}$, $P\left(\mathrm{TST}^{-} \mid \mathrm{LTBI}^{-}\right)$is the specificity of TST, and $P\left(\mathrm{QFT}^{-} \mid \mathrm{LTBI}^{-}\right)$is the specificity of $\mathrm{QFT}$ 
The model of equation (1) was used to predict the following probabilities:

p12[2]<-pi*(sens $1 *(1-$ sens 2$))+(1-$ pi $) *((1-$ spec 1$)$ *spec2)

$$
\begin{aligned}
P\left(\mathrm{LTBI}^{+} \mid \mathrm{TST}^{+}, \mathrm{QFT}^{+}\right) & =\frac{P\left(\mathrm{LTBI}^{+}\right) P\left(\mathrm{TST}^{+} \mid \mathrm{LTBI}^{+}\right) P\left(\mathrm{QFT}^{+} \mid \mathrm{LTBI}^{+}\right)}{\pi_{11}} \\
P\left(\mathrm{LTBI}^{+} \mid \mathrm{TST}^{+}, \mathrm{QFT}^{-}\right) & =\frac{P\left(\mathrm{LTBI}^{+}\right) P\left(\mathrm{TST}^{+} \mid \mathrm{LTBI}^{+}\right)\left[1-P\left(\mathrm{QFT}^{+} \mid \mathrm{LTBI}^{+}\right)\right]}{\pi_{10}} \\
P\left(\mathrm{LTBI}^{+} \mid \mathrm{TST}^{-}, \mathrm{QFT}^{+}\right) & =\frac{P\left(\mathrm{LTBI}^{+}\right)\left[1-P\left(\mathrm{TST}^{+} \mid \mathrm{LTBI}^{+}\right)\right] P\left(\mathrm{QFT}^{+} \mid \mathrm{LTBI}^{+}\right)}{\pi_{01}} \\
P\left(\mathrm{LTBI}^{+} \mid \mathrm{TST}^{-}, \mathrm{QFT}^{-}\right) & =\frac{P\left(\mathrm{LTBI}^{+}\right)\left[1-P\left(\mathrm{TST}^{+} \mid \mathrm{LTBI}^{+}\right)\right]\left[1-P\left(\mathrm{QFT}^{+} \mid \mathrm{LTBI}^{+}\right)\right]}{\pi_{00}}
\end{aligned}
$$

\section{OpenBUGS code}

model.Medellín

\{

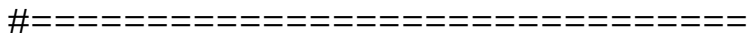

\# Observed values of multinomial random variable measuring

\# joint results of two dichotomous diagnostic tests

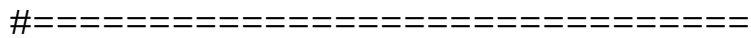

$\mathrm{t} 12[1]<-\mathrm{n} 11$

$\mathrm{t} 12[2]<-\mathrm{n} 10$

$\mathrm{t} 12[3]<-\mathrm{n} 01$

$\mathrm{t} 12[4]<-\mathrm{n} 00$

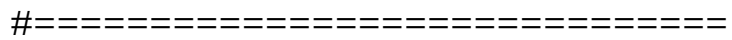

\# likelihood of observed data

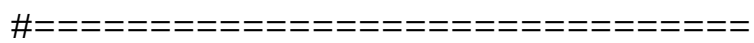
$\mathrm{t} 12[1: 4] \sim \operatorname{dmulti}(\mathrm{p} 12[1: 4], \mathrm{N})$

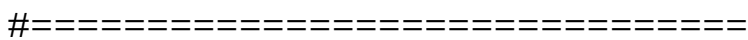

\# probabilities of observing different crossclassifications of two dichotomous diagnostic tests \#==ニ==ニ=ニ=ニ==ニ=ニ=ニ=ニ=ニ=ニ==ニ=ニ== p12[1]<-pi*(sens $1 * \operatorname{sens} 2)+(1-$ pi $) *((1-$ spec 1$) *$

$(1-$ spec2))

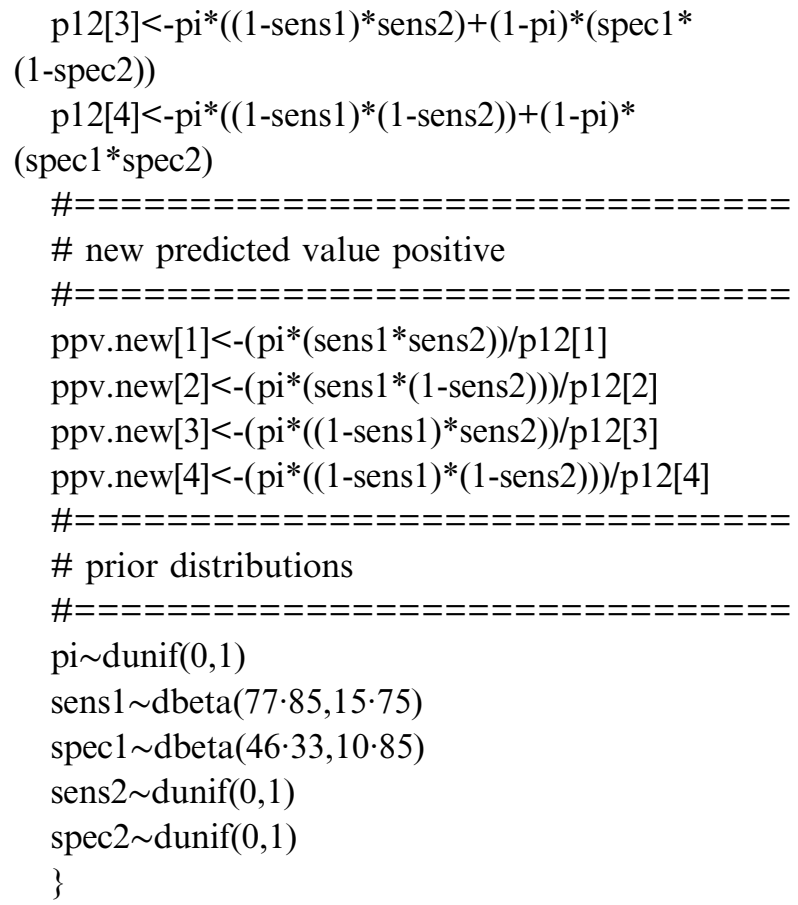

